\title{
High-Reynolds-number Batchelor-model asymptotics of a flow past an aerofoil with a vortex trapped in a cavity
}

\author{
By A. V. BUNYAKIN ${ }^{1}$, S. I. CHERNYSHENKO ${ }^{2} \dagger$ \\ AND G. Yu. STEPANOV \\ ${ }^{1}$ Department of Applied Mathematics, Krasnodar Polytechnic Institute, 350072 Krasnodar, Russia \\ ${ }^{2}$ Institute of Mechanics, Moscow University, 119899 Moscow, Russia
}

(Received 18 November 1996 and in revised form 23 October 1997)

Steady incompressible flow past an aerofoil with a cavity in its upper surface is considered. The aerofoil and cavity shape is constructed to ensure that the highReynolds-number asymptotics of the flow is described by the Batchelor model.

\section{Introduction}

The purpose of this study is twofold: to construct a non-trivial steady flow of an incompressible fluid such that its high-Reynolds-number asymptotic structure is described by the Batchelor model, and to obtain (at least) a preliminary understanding of how to design an aerofoil with a vortex cell (or a trapped vortex) contained within it in order to enhance its aerodynamic characteristics.

The famous Batchelor (1956) model was proposed as a hypothetical structure of the high-Reynolds-number limit of a steady flow past a bluff body. The true structure was found much later (Chernyshenko 1988) and turned out to be different. However, there are many papers describing calculations of various vortex-potential flows, that is inviscid flows formally corresponding to the Batchelor model, but with parameters prescribed arbitrarily instead of being determined by analyzing the viscous flow in the limit as $R e \rightarrow \infty$. So far, however, there have been no rigorous examples of non-trivial flows with the high-Reynolds-number asymptotics of the Batchelor-model type. Such an example, apart from its beauty, would be useful for theoretical fluid dynamics because it would improve our understanding of separated and recirculating flows.

The idea of using trapped vortices for enhancing the performance of aerofoils and other devices is much less well known within the academic community than the Batchelor model (and vice versa within the engineering community, see the review of Wu \& Wu 1992). This idea is illustrated by figure 1. If the aerofoil in the figure has no cavity then the dividing streamline is just a section of the rigid wall but then the boundary layer would separate on this section, resulting in a massive eddy with the usual consequences for the performance characteristics. However, this does not happen because there is a vortex cell. We may say that the dividing streamline effectively acts as a moving wall preventing the separation. At first glance it seems that a practical application of this idea is impossible because the flow shown in figure 1 is unstable: a vortex forming in the cavity will be (periodically or chaotically) shed downstream, with a new vortex generated in its stead. Such a behaviour agrees with the current understanding of

$\dagger$ Author to whom correspondence should be addressed. 


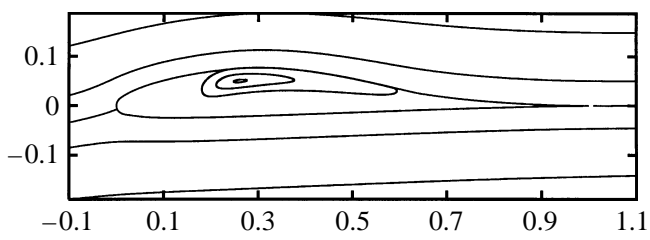

FIGURE 1. Airfoil with a trapped vortex. (Vortex-potential flow streamlines are shown. Angle of incidence 0.06 , eddy vorticity -20 , Bernoulli-constant jump 0.53.)

fluid flow stability and is observed in most experiments. There are, however, a few experiments supporting this idea (Wu \& Wu 1992), including flight tests in which the lift coefficient was reportedly four times the value characteristic for wings of conventional design, while the lift-to-drag ratio was the same. However, an attempt to reproduce this result in a wind tunnel was not successful: the flow was unstable (Kruppa 1977).

Recently successful flight tests of a model of the aircraft EKIP, designed by L. Shchukin (Savitsky et al. 1995), were performed. The body of this aircraft has the shape of a thick wing (thicker than in figure 1) with four vortex cells (cavities) on the downstream portion of the upper surface. The cells are small compared with the wing chord but much bigger than the thickness of the oncoming turbulent boundary layer, so that the flow structure may be expected to be similar to that considered in the present paper. Unlike our analysis, in Shchukin's aircraft there are central bodies inside the cells, so that the flow region inside a cavity forms a ring. Probably, this makes the flow more stable with respect to large-scale vortex shedding. Suction is used to prevent secondary separation in the downstream cells.

Generally, most experiments on trapped vortices have been unsuccessful, so to utilize this idea the shape of the aerofoil and the cavity must be very carefully chosen. On the other hand, as the high-Reynolds-number asymptotics of a flow past a bluff body is usually not of the Batchelor-model type, to construct a flow with the limit as $R e \rightarrow \infty$ of that type, the body shape must also be carefully chosen. The similarity between the two problems becomes even more evident if we notice that the general structure of a flow with a trapped vortex corresponds to the Batchelor model. This explains why we have chosen a flow with a trapped vortex as the flow for which we attempt to construct the Batchelor-model-type asymptotics. It is important to note that our approach is an inverse one: instead of looking for the asymptotics of a flow past a given body, we construct the body for a given asymptotic structure of the flow.

The following analysis is restricted to the limit as $R e \rightarrow \infty$ of steady solutions of the Navier-Stokes equations describing a plane flow of incompressible fluid. The relationship between the results obtained and realistic turbulent flows is discussed in $\S 4$ only. We consider the case of a tangential injection from the aerofoil into the cavity, because if no injection or suction is used to prevent secondary separation in the cavity then the airfoil with a trapped vortex has to be quite thin, and the cavity small and almost circular (see below), and, hence, not good for illustrative purposes.

\section{Asymptotic theory}

\subsection{Problem formulation}

Let $\boldsymbol{u}=\boldsymbol{u}(\boldsymbol{x}, R e), p=p(\boldsymbol{x}, R e)$ be a solution of the following boundary value problem for the steady Navier-Stokes equations:

$$
\left.\begin{array}{c}
\boldsymbol{u} \cdot \nabla \boldsymbol{u}=-\nabla p+(1 / R e) \nabla^{2} \boldsymbol{u}, \\
\nabla \cdot \boldsymbol{u}=0, \\
\left.\boldsymbol{u}\right|_{\Gamma}=\boldsymbol{u}_{w},\left.\quad \boldsymbol{u}\right|_{|x| \rightarrow \infty}=\boldsymbol{u}_{\infty},
\end{array}\right\}
$$


where $\Gamma$ is the body surface, $\boldsymbol{u}_{w}$ is the injection velocity, and $R e$ is the Reynolds number. Then the problem is to find a shape of the body surface $\Gamma$ (aerofoil with a cavity shape) and, optionally, the injection velocity distribution, such that the limit of $\boldsymbol{u}(\boldsymbol{x}, R e)$, and $p(\boldsymbol{x}, R e)$ as $R e \rightarrow \infty$ is described by the Batchelor model. The flow structure itself should also be found in this limit.

This means that as $R e \rightarrow \infty$ the flow must be inviscid apart from certain regions, at least one of whose dimensions tends to zero as $R e \rightarrow \infty$ (such as in a boundary layer). Moreover, outside the region of closed streamlines the flow must be potential, whilst, by the Prandtl-Batchelor theorem, the vorticity must be constant inside the closed streamline region. There may be a discontinuity of the Bernoulli constant across the boundary of this region. Let $\Psi$ and $\omega$ be a stream function and vorticity respectively. Then the limiting flow is a solution of the following problem:

$$
\left.\begin{array}{cl}
\nabla^{2} \Psi=0 & \text { outside the eddy, } \\
\nabla^{2} \Psi=-\omega=\mathrm{const} & \text { inside the eddy, } \\
\left.(\nabla \Psi)^{2}\right|_{\Psi=0+}-\left.(\nabla \Psi)^{2}\right|_{\Psi=0-}=2[H]=\mathrm{const} & \text { along the eddy boundary, } \\
\left.\Psi\right|_{\Gamma}=0, \quad \nabla \times\left.(\Psi \boldsymbol{k})\right|_{|x| \rightarrow \infty}=\boldsymbol{u}_{\infty} . &
\end{array}\right\}
$$

Here, $\boldsymbol{k}$ is a unit vector perpendicular to the flow plane. Note that there is no injection in this problem since for our purposes it is sufficient to inject fluid inside the boundary layer, with the flow rate of the same order of magnitude as the flow rate in the boundary layer, that is tending to zero as $R e \rightarrow \infty$. Naturally, such an injection does not affect the formulation of the problem for the external inviscid flow.

\subsection{Basic properties of vortex-potential flows}

A vortex-potential flow is an inviscid flow satisfying (2.2) whether or not it is the true high-Re limit of the corresponding viscous flow. We will consider the case when there is just one closed streamline region. Numerous studies of vortex-potential flows show that in the general case, for a given body shape, there is a two-parameter family of vortex-potential flows. This is not proved rigorously in the general case, but is rather a conclusion of many studies of particular cases, simplified formulations, and full numerical computations. Some references can be found in Bunyakin, Chernyshenko \& Stepanov (1996), which describes the method of constructing vortex-potential flows used in our study. The eddy vorticity $\omega$ and the Bernoulli constant drop across the eddy boundary $[H]$, for example, can be used as the parameters of the family. In that case the location of the separation and reattachment points can be found from the solution. It is more natural, however, to prescribe the position of the separation point, because if the body surface has a corner point then separation occurs there. With the location of the separation point fixed, there is only one free parameter. There is an essential difference between vortex-potential flows in general and the Batchelor-model flow. It is reasonable to assume that for a given problem there is only one, or at the most a finite number of limiting flows as $R e \rightarrow \infty$, but not a one- or two-parameter family. Therefore, the Batchelor-model flow is one of the vortex-potential flows which is the true limit of the viscous flow as $R e \rightarrow \infty$. Mixing notions may sometimes result in misunderstanding.

In constructing asymptotic solutions as $R e \rightarrow \infty$ the pressure distribution along the body surface plays an important role. The boundary layer may separate from a portion of the body surface with an unfavourable pressure gradient at a point not implied by the general structure of the vortex-potential flow. It appears that vortex-potential flows without a portion of adverse pressure gradient upstream of the separation point 
are rare. If the flow velocity inside the eddy equals zero at the separation point of the external flow (and this is the case if separation occurs at a corner of non-zero angle or from a smooth surface, that is unlike in figure 1) then the pressure distribution near the separation point is similar to that in a flow with a free streamline with a constant pressure, that is both the curvature of the dividing streamline and the pressure gradient tend to infinity as the separation point is approached along the streamline from downstream and upstream respectively. For flows in cavities (Herwig 1982) and for a flow past a backward-facing step (Chernyshenko 1984) it was shown that this infinite pressure gradient is unfavourable. Moreover, Chernyshenko (1984) showed that in a symmetrical vortex-potential flow past a body, the existence of a section of the body surface with an unfavourable pressure gradient upstream of a separation point is the rule rather than an exception.

It is natural to locate a vortex cell on the aerofoil in such a way that the portion of the upper wing surface with an unfavourable pressure gradient becomes a boundary of the vortex cell (as this is expected to prevent a massive separation). It seems possible to find a shape of the cavity such that the vortex-potential flow decelerating along the dividing streamline will then only accelerate along the cavity wall, so that everywhere on the rigid wall the pressure gradient will be favourable. Unfortunately, there are no such cavity shapes. Consider an inviscid flow in a closed streamline region. As the fluid density is constant, the total momentum and angular momentum of the fluid in the eddy are equal to zero and do not vary with time, although this fluid volume is under the action of pressure forces at the boundary. Therefore, the total force and the total moment of force with respect to an arbitrary point are equal to zero. This can be written in the following form:

$$
\oint p r \mathrm{~d} r=\oint p r \frac{\partial r}{\partial s} \mathrm{~d} s=0,
$$

where the integral is taken over the eddy boundary, $p$ is the pressure, and $r$ is the distance to a point $O$ (so far undefined), with respect to which the moment of force is calculated. Suppose that there is only one point of minimum pressure and one point of maximum pressure on the contour and that a circle can be drawn through these points without other intersections with the eddy contour. This is always possible for a convex contour and for many other contours of a not too complicated shape. Let $O$ be the centre of this circle and $r_{0}$ its radius. Then the product $(\partial p / \partial s)\left(r^{2}-r_{0}^{2}\right)$ does not change sign on the contour. After simple transformations we obtain

$$
\oint p r \frac{\partial r}{\partial s} \mathrm{~d} s=\oint \frac{1}{2}\left[\frac{\partial p\left(r^{2}-r_{0}^{2}\right)}{\partial s}-\left(r^{2}-r_{0}^{2}\right) \frac{\partial p}{\partial s}\right] \mathrm{d} s=-\oint \frac{\left(r^{2}-r_{0}^{2}\right)}{2} \frac{\partial p}{\partial s} \mathrm{~d} s \neq 0 .
$$

The contradiction between (2.3) and (2.4) proves that the initial assumption cannot be valid. Numerical calculations also show that there are at least two pressure minima and maxima.

\subsection{Restrictions on the position of the viscous separation point}

In an inviscid flow the position of the separation point can be prescribed arbitrarily to a certain extent. However, analysis of a viscous flow close to the separation point shows that if the inviscid flow is the high-Re limit of a viscous flow then the position of the separation point satisfies additional conditions. Such a local analysis leads to various versions of the widely known theory of viscous-inviscid interaction. Extensive information on this theory can be found in the book by Sychev et al. (1987). There are three possibilities: separation can occur at a corner point, or at a point on a 
smooth surface where the condition of a smooth separation, that is the BrillouinVillat condition, is satisfied or at an upstream-directed sharp edge. In all these cases the separation point in the viscous flow is downstream from its limiting position and moves upstream as the Reynolds number tends to infinity. Generally, in an inviscid flow upstream of the separation point $\partial p / \partial s=k\left(s_{0}\right) /\left(s_{0}-s\right)^{1 / 2}+\ldots$, where $s$ is a coordinate measured in the flow direction, and $s=s_{0}$ is the separation point. If $k\left(s_{0}\right)>0$ then the boundary layer will separate upstream from the separation point of the inviscid flow, which is clearly inconsistent. Hence, inviscid flow with $k\left(s_{0}\right)>0$ can be a high-Re limit of a viscous flow only if there is no boundary layer upstream from the separation point. This is the case if separation occurs from an upstream-directed sharp edge, as described by Sychev (1978). If $k\left(s_{0}\right)<0$ then separation can occur at a corner point (Ruban 1974, 1976) but not from a smooth surface because in the latter case the dividing streamline would penetrate the body surface. A self-consistent, local viscous structure near the separation point on a smooth surface (Sychev 1972; Smith 1977) exists only if in the inviscid flow $k\left(s_{0}\right)=0$.

Therefore, a local analysis of viscous effects reduces the number of free parameters of the vortex-potential flow either by fixing the separation point at a corner or an edge or by giving the additional condition $k\left(s_{0}\right)=0$. As the analysis resulting in these restrictions remains valid for $\omega=0$, it is sometimes said that it gives the value of $[H]$, keeping in mind, of course that in fact $\omega$ and $[H]$ can be found only simultaneously.

\subsection{Formulation of the problem of a cyclic boundary layer}

Suppose that the solution of (2.2) is known. Then the boundary layer on the body surface must be calculated. The boundary layer begins at the forward stagnation point and continues to the separation point, and on the lower surface of an aerofoil to the trailing edge. There is also a boundary layer on the cavity wall. Near the dividing streamline there is a mixing layer which is also governed by the boundarylayer equations. Finally, there is a boundary layer between the reattachment point and the trailing edge on the upper surface. In the general case the boundary-layer approximation becomes invalid near the separation and reattachment points, where new distinguished limits in the so-called turn regions must be introduced. It turns out, however, that the flows in the boundary layers pointed out above can be matched through the turn regions without analysing these regions in detail. The reason is that inside the regions with one dimension much larger than the other the boundary-layer equations remain valid, as can be checked with the heuristic principle of Kaplun (Cole 1968), so that only the distinguished limits in the regions of equal longitudinal and transverse dimensions need be considered. Let us estimate the characteristic Reynolds number in such regions. The characteristic flow rate inside these regions is the same as in the boundary layer: $Q_{t u r n} \sim R e^{-1 / 2}$ (all parameters are already non-dimensionalized with the velocity at infinity and characteristic size of the body). Correspondingly, the characteristic Reynolds number $R e_{\text {turn }} \sim U_{\text {turn }} L_{\text {turn }} R e \sim$ $Q_{\text {turn }} R e \sim R e^{1 / 2} \rightarrow \infty$. Hence, in the leading term the flow inside the turn region is effectively inviscid. Therefore, the Bernoulli theorem holds true there. If the boundarylayer equations are solved in Mises variables, using $g=u^{2} / 2+p$ as the dependent variable, where $u$ is the velocity, and the stream function $\psi$ as the independent variable then the existence of the turn regions can be just ignored (Matveeva \& Neiland 1977; Neiland \& Sychev 1966). This is of course valid also for the case shown in figure 1 when at the separation and reattachment points the body contour forms cusps. 


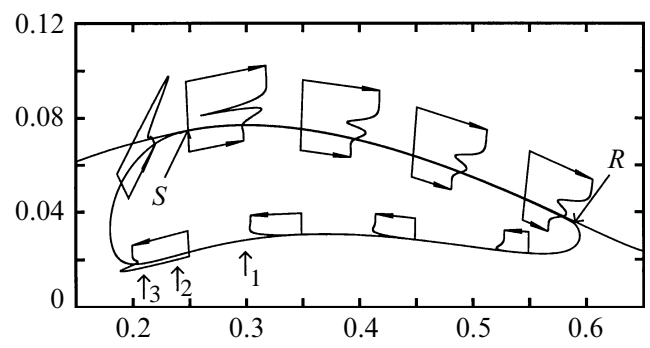

FiguRE 2. Velocity as a function of $\psi$ in the cyclic layer. The picture is stretched vertically for a better view. Velocity and stream function scales are 0.05 and 0.003 respectively. The blowing flow rate equals 0.6 at each of the points shown with arrows $(s=0.65,0.71$, and 0.74$)$. The blowing stagnation pressure is 0.77 (at infinity the velocity is equal to 1 and the stagnation pressure is zero).

The boundary layer between the forward stagnation point and the separation point can be calculated in the usual way. This gives the velocity, and, hence, the $g=g_{0}(\psi)$ profile immediately upstream of the separation point. Let us introduce a coordinate system $s, n$ with the origin at the separation point $S$, with $s$ measured clockwise along the dividing streamline and the cavity wall, and $n$ increasing outward from the eddy (figure 2). We denote the reattachment point coordinate as $s_{R}$. The separation point is at $s=0$ and $s_{S}$ is the length of the contour $S R 123 S$ (figure 2). Points of tangential injection 1,2 , and 3 are at $s_{1}, s_{2}$, and $s_{3}$. The injection flow rate $R e^{-1 / 2} Q$ and the stagnation pressure $g_{I}$ of the injected fluid are assumed to be the same for all the injection points. (As mentioned above, the injection is introduced in this work in order to improve the external appearance of the aerofoil obtained. For this reason no systematic study of the influence of these parameters was made.) We introduce a stream function $\psi$ scaled to be of order one in a boundary layer, so that the longitudinal velocity $u=R e^{-1 / 2} \partial \psi / \partial n$. Then the boundary value problem for the cyclic boundary layer can be written in the following form:

$$
\frac{\partial g}{\partial s}=u \frac{\partial^{2} g}{\partial \psi^{2}}
$$

matching with oncoming layer,

$$
s=0, \psi>3 Q, g(0, \psi)=g_{0}(\psi),
$$

periodicity condition,

$$
s=0, \psi<3 Q, g(0, \psi)=g\left(s_{S}, \psi\right),
$$

no-slip condition,

$$
\begin{array}{lr}
s_{R}<s<s_{1}, & g(s, 0)=p(s), \\
s_{1}<s<s_{2}, & g(s, Q)=p(s), \\
s_{2}<s<s_{3}, & g(s, 2 Q)=p(s), \\
s_{3}<s<s_{S}, & g(s, 3 Q)=p(s),
\end{array}
$$

injection condition,

$$
\begin{gathered}
\frac{\partial g}{\partial s}=u \frac{\partial^{2} g}{\partial \psi^{2}}, \\
\text { matching with oncoming layer, } \\
s=0, \psi>3 Q, g(0, \psi)=g_{0}(\psi), \\
\text { periodicity condition, } \\
s=0, \psi<3 Q, g(0, \psi)=g\left(s_{S}, \psi\right), \\
\text { no-slip condition, } \\
s_{R}<s<s_{1}, \quad g(s, 0)=p(s), \\
s_{1}<s<s_{2}, \quad g(s, Q)=p(s), \\
s_{2}<s<s_{3}, \quad g(s, 2 Q)=p(s), \\
s_{3}<s<s_{S}, \quad g(s, 3 Q)=p(s), \\
\text { injection condition, } \\
s=s_{i},(i-1) Q<\psi<i Q, g\left(s_{i}, \psi\right)=g_{I}, i=1,2,3, \\
\text { uniqueness condition, } \\
\lim _{\psi \rightarrow-\infty} g(s, \psi)<\infty .
\end{gathered}
$$

We denote $G_{\infty}=\lim _{\psi \rightarrow-\infty} g(s, \psi)$. From (2.5) it follows that $G_{\infty}$ does not depend on $s$. 


\subsection{Properties of the boundary value problem}

Boundary value problems of this type are well studied (Chernyshenko 1982, 1984, 1991), although rigorous results were obtained for a heat diffusion equation only (Kolosov \& Shifrin 1975). If the solution of (2.5) with $G_{\infty}<\infty$ exists for a given pressure distribution then it will be unique. Importantly, for uniqueness $G_{\infty}$ need not be prescribed, but instead can be determined as part of the solution. This closes the problem of constructing the entire asymptotic structure. Indeed, the matching condition between the cyclic boundary layer and the vortex-potential flow requires $G_{\infty}$ to be equal to the stagnation pressure $p_{*}$ on the outermost closed streamline in the inviscid flow $\left(p_{*}+[H]\right.$ is the stagnation pressure in the potential flow outside of the eddy). For a given body shape the vortex-potential flow and, hence, the pressure distribution are uniquely determined by two parameters $[H]$ and $\omega$. Therefore, for given values of these parameters, it is possible to solve (2.2) and then (2.5) to obtain $G_{\infty}=G_{\infty}\left([H], \omega, Q, g_{I}\right)$. The matching condition takes the form

$$
G_{\infty}\left([H], \omega, Q, g_{I}\right)=p_{*} .
$$

As has already been explained, if the flow satisfies a local condition at the separation point, whether it is the Brillouin-Villat condition or the fixed position condition, $[H]$ and $\omega$ are not independent. Hence, for $Q$ and $g_{I}$ given, (2.6) is an equation with just one unknown. The fact that analysis of a cyclic layer makes it possible to determine one parameter was demonstrated with a simple example by Squire as early as 1956.

It is, however, possible that (2.5) has no solution at all. If the unfavourable pressure gradient in a cyclic layer is sufficiently large, then a singularity appears in the solution indicating that there is a secondary separation. In particular, it was discovered in numerical calculations and then proved theoretically (Chernyshenko 1991) that a secondary separation is inevitable if the inviscid flow inside the eddy has a stagnation points on its boundary, and even in cases when this takes place at the reattachment point only. The idea of the proof involves the fact that $g(s, \psi)$ approaches $G_{\infty}$ with decaying oscillations as $\psi \rightarrow-\infty$, so that for each $s$ there is a value of $\psi$ for which the velocity in the cyclic layer is strictly less that its value at $\psi=-\infty$. Hence, if that value at infinity approaches zero, as is the case at a stagnation point, then somewhere upstream of it the velocity will become zero at some point inside the layer, thus developing a singularity. This means that the external inviscid flow should be modified by introducing another separation region. However, the same reasoning applies to the boundary layer surrounding this secondary eddy, and so on. Therefore, there should be an infinite number of nested eddies, although such a flow cannot be described by the Batchelor model. Note that the secondary separation described by this mechanism appears within the bulk of the boundary layer but not at a wall. Of course, an unfavourable pressure gradient on the cavity wall can also result in a secondary separation in the usual way.

\subsection{Summary of requirements for the body shape}

Therefore, to design a body the flow past which has a Batchelor-model asymptotics, it is necessary to:

1. choose the body shape so that there is no strong unfavourable pressure gradient upstream of the separation point, in order to prevent premature separation;

2. provide for separation and reattachment at cusps, in order to prevent secondary separation in the bulk of the cyclic layer near the stagnation points of the inviscid flow; 
3. make the cavity almost circular or use other means (for example, blowing or suction) in order to prevent secondary separation from the cavity wall.

\section{Numerical calculations}

\subsection{Notes on numerical calculation of cyclic boundary layers}

A detailed description of the particular computational method is outside the scope of this paper.

Most often the characteristic thickness of an ordinary boundary layer grows monotonically downstream. However, in the case of a cyclic layer this thickness is a periodic function of the longitudinal coordinate. As the velocity of the external inviscid flow decreases, the cyclic layer thickness grows and vice versa. These variations may be quite large if the cyclic layer is on the verge of separation, which is almost always the case. For this reason it is much more convenient to perform a calculation in the Mises variables $s$ and $\psi$. Then variation of the external flow velocity does not cause large variations of the thickness, because in these variables the characteristic thickness is in fact the characteristic flow rate in the layer. However, the advantage of using a computational domain of constant width is counterbalanced by increased requirements on the speed and memory size of the computer, for the following reasons.

Some caution is needed for the finite-difference approximation of equations (2.5) in the case when the boundary layer is bounded by a wall where the no-slip condition is imposed. In this case $g$ as a function of $\psi$ is singular at the wall, and a usual finite-difference approximation of the second derivative is invalid. We overcame this difficulty by representing $g(s, \psi)$ on the grid $s_{i}, \psi_{j}$, but approximating derivatives on the corresponding grid in the $(s, n)$-plane, where $n$ is the coordinate normal to the wall. In the case of constant steps in $s$ and $\psi\left(\Delta \psi=\psi_{j+1}-\psi_{j}, \Delta s=s_{i+1}-s_{i}\right)$ and implicit approximation of the second derivative this approach gives the following finite-difference equation:

$$
\frac{g_{i+1, j}-g_{i, j}}{\Delta s}=\frac{\left(u_{i, j+1}+u_{i, j}\right)\left(u_{i, j}+u_{i, j-1}\right)}{u_{i, j+1}+2 u_{i, j}+u_{i, j-1}} \frac{g_{i+1, j+1}-2 g_{i+1, j}+g_{i+1, j-1}}{(\Delta \psi)^{2}} .
$$

This equation was used to obtain the numerical results presented in this paper. In other calculations (not given here) we have used variations to this approach for the case of a non-uniform grid and an explicit-implicit scheme of higher order in $\Delta s$.

For smooth solutions equation (3.1) gives second-order accuracy in $\Delta \psi$ and first in $\Delta s$. However, near the wall and the points where derivatives are not continuous (in a cyclic layer these are the separation, reattachment and injection points, because the boundary conditions are not continuous there) the order of approximation is reduced. In practice (see the analysis of the approximation of the particular computation below) the order of approximation is $(\Delta \psi)^{1 / 2},(\Delta s)^{1 / 2}$. As there are several reasons for the reduction in the order of approximation, improving it requires more complicated schemes. Although this decreases the time of a single computation, time spent on developing a program and determining the optimal parameters (for example, of a non-uniform grid) increases, so that on the whole the simplest scheme (3.1) turns out to be the most advantageous.

Equation (3.1) can be solved by marching methods stepping in $s$. However, the problem (2.5) does not contain all the necessary initial conditions. Instead there is a periodicity condition at $s=0, s=s_{S}$. For this reason iteration is necessary: prescribing $g(0, \psi)$, we proceed with a marching method to $s=s_{S}$, use the calculated 
profile of $g\left(s_{S}, \psi\right)$ as the new approximation for $g(0, \psi)$ and repeat this procedure until convergence. This procedure can be considered as a result of a periodic continuation of the computational domain to infinity downstream. Convergence then means that the solution should become independent of the initial conditions. It is clear now that if the computational domain were infinite not only in $s$, but also in the direction of decreasing $\psi$, as is the case in (2.5), then there would be no convergence, or, more exactly, the convergence would be non-uniform: for a finite $\psi$ given, $g(s, \psi)$ would approach the solution of (2.5) as $s \rightarrow \infty$, but for a finite $s$ given $g(s, \psi)$ would not approach the solution of (2.5) as $\psi \rightarrow-\infty$ but would tend to a value determined by the upstream initial conditions. Therefore, the larger the computational domain the larger the number of iterations needed to achieve convergence. On the other hand decreasing the width of the computational domain increases the error caused by the replacement of an infinite region with a finite one. For this reason iterations in a cyclic boundary layer converge slowly, and the larger the computational domain the slower the convergence. As a result, the iteration should not be stopped by the criterion of a small difference in the solution on two successive iterations. Instead, special criteria valid for slowly converging iterations must be used.

The width of the computational domain is determined, as usual, through test calculations, and is increased until the solution ceases to vary within the prescribed accuracy. Here, however, the following should be taken into account. The finitedifference scheme (3.1) is non-conservative. In general, it is difficult to construct a finite-difference scheme in Mises variables which would be conservative with respect to momentum. Hence, the approximation error may manifest itself as some additional force with a non-zero total effect in the boundary layer. In order to understand the result of this, assume for simplicity that this additional force is proportional to the velocity in the boundary layer. Then, taking into account the additional term, the principal equation in (2.5) takes the form

$$
\frac{\partial g}{\partial s}=u \frac{\partial^{2} g}{\partial \psi^{2}}-C_{1} u .
$$

The value of $C_{1}$ tends to zero as the grid size tends to zero. In the general case, as $\psi \rightarrow-\infty$ the periodic solution of this equation takes the form

$$
g(s, \psi) \rightarrow G_{\infty}+C_{2} \psi+C_{1} \psi^{2} / 2 .
$$

The constraint $\lim _{\psi \rightarrow-\infty} g(s, \psi)<\infty$ needed for the uniqueness of the solution means that in the solution sought $C_{2}=0$. In a numerical computation this can be achieved by imposing $\partial g / \partial \psi=0$ at the base of the computational domain. If convergence is achieved, $G_{\infty}$ is determined to be equal to the value of $g(s, \psi)$ at the base of the computational domain, and the condition that this value is equal to the corresponding value on the outer-most closed streamline of the inviscid flow can be used, as described in previous sections, for determining a parameter of the vortex-potential flow family. If, however, $C_{1} \neq 0$ then $g(s, \psi)$ at the base of the computational domain will not tend to a finite limit as the width of the domain tends to infinity. Therefore, before determining the width of the computational domain the convergence with respect to the grid size should be achieved (that is $C_{1}$ should be made sufficiently small).

Instead of imposing $\partial g / \partial \psi=0$ at the base of the computational domain it is better to prescribe $g$ to be equal to the corresponding value in the inviscid flow and to use the condition $\partial g / \partial \psi=0$ to determine the parameter of the vortex-potential flow 


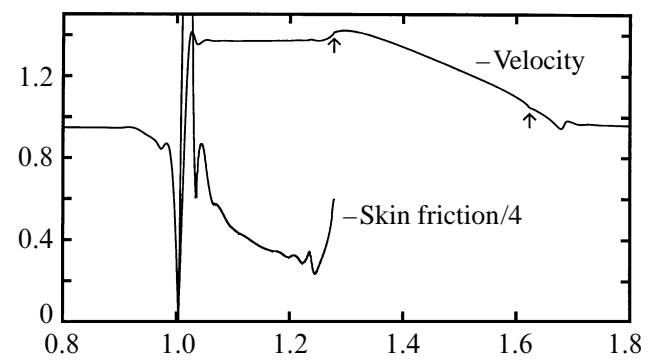

FIGURE 3. Skin friction and potential flow velocity. Near the trailing edge and over most of the lower surface (not shown) the velocity is constant. The cavity is between the arrows. The abscissa is measured clockwise from the trailing edge.

family. The rigid boundary condition makes the iterations in a cyclic layer converge faster.

\subsection{An example of a calculation}

An aerofoil is designed in several steps.

Step 1. The calculation starts from determining the aerofoil and dividing streamline shape. This was carried out using the code POLET developed in the Institute of Mathematics and Mechanics of the Kasan' State University (Russia). This code solves the inverse problem of a potential flow past an aerofoil. It allows us to prescribe a desirable velocity distribution and to obtain an aerofoil with the velocity distribution as close to that prescribed as possible. Moreover, it is possible to obtain exactly the prescribed velocity distribution on a portion of the aerofoil. Using this code we constructed an aerofoil for which the velocity quickly grows from the stagnation point to certain values on the upper and lower surface. Over the rest of the lower surface it then remains constant. On the upper surface the velocity is also constant upstream of some point (which then will be converted into a separation point by introducing a cavity), then smoothly decreases to the value of the velocity on the lower surface and remains constant over the rest of the upper surface. This velocity distribution was already close to the final distribution shown in figure 3. This approach ensures that there will be no separation outside the portion where the cavity will be introduced. The code POLET gives the aerofoil shape as an array of points. This representation was used for further calculations, with the aerofoil shape between points described by a cubic spline, as in Bunyakin et al. (1996).

Step 2. The portion of the aerofoil surface at which the pressure gradient is unfavourable is fitted with a portion of parabola, which will later become the dividing streamline. A parabola can be described by an explicit expression, and this is necessary for further construction of the cavity. The end points of this portion now become corner points of the aerofoil contour, although the angles are quite close to $2 \pi$. Hence, the oncoming boundary layer will separate upstream of the first of these points. Moreover, when the cavity is constructed, instead of cusps at the beginning and the end of the cavity there would be corner points, which are undesirable (see above). For this reason the aerofoil shape was further modified just outside that portion of the parabola: the nearest points determining the aerofoil shape were shifted manually in such a way that the aerofoil contour smoothly transforms into the parabola. This prevents separation, but results in small oscillations of higher derivatives of the cubic spline which describes the contour between the fixed points. Eventually, these 


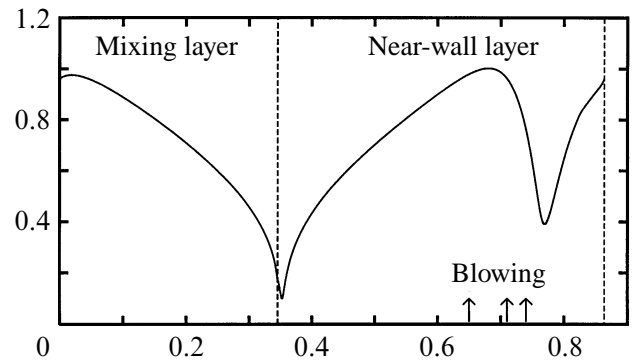

FIGURE 4. Inviscid flow velocity inside the cavity at the edge of the viscous layer.

oscillations lead to the velocity and friction oscillations in figure 3. Note that these oscillations do not result from a numerical or approximation error. The accuracy of the results presented here ensures that the error in the plots is less then the thickness of the lines. The potential flow past the obtained aerofoil was then calculated. The method described by Kuethe \& Chow (1986) was used with two modifications. First, the Gauss method was used to solve the system of linear equations instead of the Cramer rule and, second, we used Tikhonov regularization (Tikhonov et al. 1990) to enable the program to calculate flows past aerofoils with a sharp trailing edge. Then the boundary layer between the stagnation point and the beginning of the cavity was calculated. Figure 3 shows the results for the final version of the aerofoil and dividing streamline shape. It can be seen that there is no premature separation.

Step 3. For given values of the drop in the Bernoulli constant across the dividing streamline $[H]$ and the vorticity $\omega$ in the eddy, it is possible to find a cavity such that the corresponding vortex-potential flow will coincide outside the eddy with the already calculated potential flow outside. For this purpose, a known rotational motion with the same $\omega$, for example a rigid-body rotation, is subtracted from the vortex-potential flow, and the remaining potential flow is determined by analytic continuation from the dividing streamline. The method is described in detail by Bunyakin et al. (1996). Varying $[H]$ and $\omega$ makes it possible to obtain suitable velocity distributions on the cavity wall. The final version is shown in figure 4 . This velocity distribution has two minima and, accordingly, two portions with unfavourable pressure gradients. The first is immediately downstream from the reattachment point and is very short. For this reason, although the skin friction drops sharply here, there is no secondary separation. If $\omega$ and $[H]$ are changed so that this portion is longer then there could be a separation. On the second portion with unfavourable pressure gradient, the secondary separation was prevented by tangential injection.

The final aerofoil and cavity shape and the streamlines of the vortex-potential flow are shown in figure 1.

Step 4. Finally, the cyclic boundary layer is calculated. As mentioned, in this calculation we prescribe $g$, obtained from the calculation of the vortex-potential flow (that is $p_{*}$ ) as the boundary condition at the boundary of the computational domain, which is turned toward the cavity (or, equivalently, at this boundary the velocity in the boundary layer is prescribed to be equal to the velocity of the inviscid flow in the cavity when this boundary is approached from within the cavity). Then as a result of the calculation $F=\partial g / \partial \psi$ on this boundary is determined. Now, the matching condition (2.6) implies that $F=0$ in a valid asymptotic limit as $R e \rightarrow \infty$. As $F=F\left([H], \omega, Q, g_{I}\right)$ we can fix any three of its arguments and find the fourth by solving numerically the equation $F=0$. In our case it is convenient to fix $[H]$ 


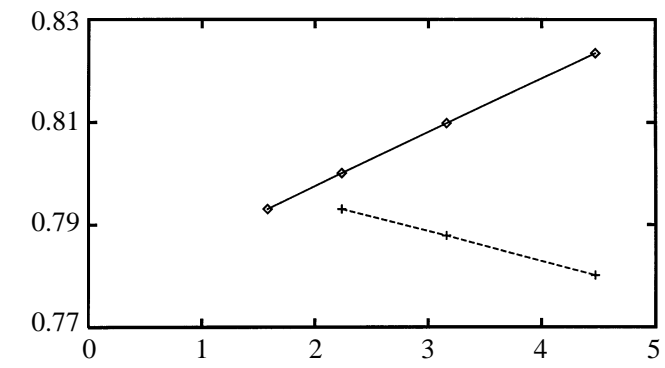

Figure 5. Accuracy analysis: $g_{I}$ as a function of $100 M^{-1 / 2}$ for $\Delta \psi=$ const (solid) and of $100(\Delta \psi)^{1 / 2}$ for $M=$ const (dashed).

and $\omega$ because then we need not recalculate the vortex-potential flow when solving the equation $F=0$, and $Q$ because this determines the shape of the computational domain in the cyclic boundary layer.

Steps 3 and 4 may be unsuccessful: step 3 for the reasons explained in Bunyakin et al. (1996) and step 4 because of a secondary separation. By trial and error, varying the initial velocity distribution in step 1 , the positions of the separation and reattachment points in step 2 , and $[H], \omega, s_{1}, s_{2}, s_{3}$, and $Q$, we finally obtained the self-consistent result, presented in the figures in this paper.

Calculations of the cyclic layer were performed with various grids and computational domains in order to ensure that sufficient accuracy was obtained. The grid was uniform in $\psi$ and $s$. The base of the computational domain was at $\psi=-3$. The top was at $\psi=7$ in the mixing layer (with $\psi=0$ corresponding to the reattaching streamline), and at $\psi=0$ in the boundary layer on the cavity wall before the first injection point, and then the height of the top was increased by $Q=0.6$ at each injection point. The number of steps in $s$ was $2 M$ in the mixing layer, $2 M$ between reattachment and the first injection point, and $M$ between injection points and between the last injection point and the separation point. The most accurate of our calculations was made with $M=4000$ and $\Delta \psi=0.0005$. Figure 5 shows the dependence of the most sensitive quantity, namely the stagnation pressure $g_{I}$ of the injected fluid, on $M^{-1 / 2}$ and $(\Delta \psi)^{1 / 2}$. The plots show a linear dependence giving the order of accuracy, which may be approximated with the formula

$$
g_{I}=0.800+1.07 M^{-1 / 2}-0.557(\Delta \psi)^{1 / 2},
$$

thus giving the value $g_{I}=0.80$ as the final result. We also tried to extrapolate similarly the other results presented in the plots throughout this paper but it turned out that the extrapolated plots were indistinguishable from those obtained from our highest-accuracy calculation. Other parameters prescribed or obtained are: angle of incidence 0.06, $[H]=0.53, \omega=-20, s_{R}=0.346, s_{S}=0.863, s_{1}=0.65, s_{2}=0.71$, $s_{3}=0.74$, and $Q=0.6$.

Figures 6 and 7 show the velocity profiles at $s=0,0.1,0.2,0.3,0.4,0.5,0.6$, 0.7, 0.8 plotted against the transverse coordinate in the cyclic layer (unlike figure 2 where these were plotted against $\psi$ ). It is noticeable that in the bulk of the layer the low-velocity areas are in a sense persistent and expand far downstream, after departing from the wall at separation and injection points. As a result, if the vortexpotential flow has regions of strong adverse pressure gradient, then a singularity appears within a cyclic layer. For this reason it seems that for preventing secondary separation suction may be more efficient than injection, because suction would remove 


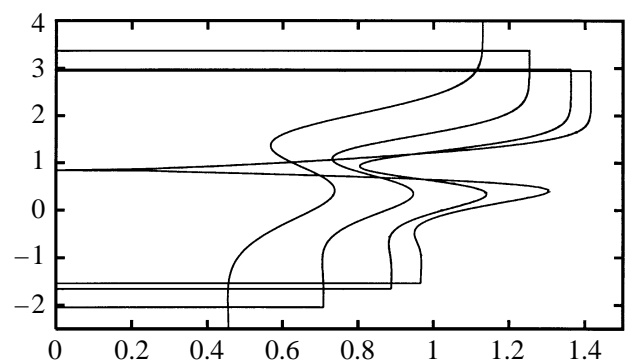

FIGURE 6. Velocity profiles in the mixing layer at $s=0,0.1,0.2,0.3$. External flow decelerates.

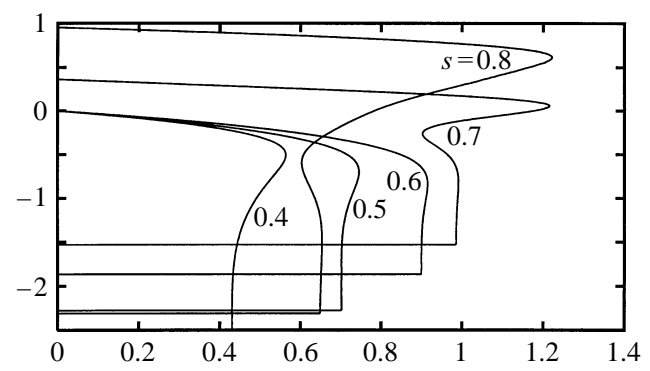

FIGURE 7. Velocity profiles in the boundary layer on the cavity wall.

the low-velocity fluid from the flow. Although no systematic study of the influence of numerous parameters in this problem was made, our experience suggests that Batchelor-model-type asymptotics is a proper one only in a very limited region of the parametric (initial aerofoil shape, $[H], \omega, s_{1}, s_{2}, s_{3}$, and $Q$ ) space.

\section{Concluding remarks}

There are two different mechanisms by which a trapped vortex may inhibit separation. Suppose that a cavity is located upstream of the point where the flow separates in the absence of the cavity. Then the velocity profile in the boundary layer will become more filled above the cavity. In a sense this is equivalent to a downstream shift of the beginning of the boundary layer which naturally shifts the separation point downstream too. To implement this mechanism it is not necessary to locate the cavity in the area of an unfavourable pressure gradient.

This shows that it is possible in principle to design an aerofoil with a trapped vortex without blowing or suction preventing the secondary separation. Let us first take an ordinary aerofoil the flow past which does not separate. Then let us increase the angle of incidence to a slightly supercritical value, so that the flow is separated. Then, by adding a small almost circular cavity with only a very small opening (very short mixing layer) near the pressure minimum on the aerofoil surface upstream of the marginal separation point it will be possible to prevent separation provided that the overshoot of the angle of incidence over the critical value is sufficiently small. In this case the pressure variation in the mixing layer, and, hence, in the cavity, may be quite small, so that no secondary separation in the cavity should be expected.

The second mechanism is a redistribution of the unfavourable pressure gradient on a portion of aerofoil surface over a portion of the cavity surface. In that case the 
cavity is located in such a way that the fluid decelerates along the dividing streamline. It should be noted that although a reversed flow is less likely to develop in a mixing layer than in a near-wall layer nevertheless this cannot be always excluded, as is clearly indicated by figure 1 , where the velocity minimum decreases as $s$ increases. Also, as was proved in the end of $\S 2.2$, there should be portions of the cavity wall with unfavourable pressure gradient so that suction or injection are likely to be necessary to prevent secondary separation inside the cavity. Nevertheless, additional freedom in the possible distribution of unfavourable pressure gradients over the walls may be used to decrease the intensity of suction or injection. However, an assessment of the practical effectiveness of these two mechanisms and their comparison with other methods of preventing separation lay outside the scope of the present paper. In fact, before such assessment can become possible methods of calculating turbulent flows of this type should be developed.

The results presented here describe a non-trivial example of a steady flow the high-Re asymptotics of which is described by the Batchelor model. They show in detail the mechanisms and existence of such flows and demonstrate that such flows are quite rare. Results obtained with asymptotic methods on the basis of the full Navier-Stokes equations have the advantage of assured self-consistency and objectiveness. In other words, due to the formal nature of the method there can be no internal contradiction in the flow structure obtained, and this structure is independent of the researcher's preferences. This would not be the case if turbulence models were introduced (and rejected) in the process of solution because the selection of the turbulence model could be affected by the researcher's intuitive idea about the flow structure. However, as soon as a laminar asymptotic structure is found, certain steps can be taken toward the description of the corresponding turbulent flow. Suppose that a realistic turbulent flow with a trapped vortex does not exhibit a large-scale unsteadiness, either due to specific geometry (as it probably is in the case of the EKIP vehicle) or special measures such as imposed high-frequency oscillations or both (see again Wu \& Wu 1992). Then it may be possible to construct the flow description by substituting a turbulent cyclic boundary layer for the laminar one in our theory. This depends on whether the flow inside a trapped vortex (that is stable on a large scale) is effectively inviscid. Although some indications supporting this possibility can be found in experiments (Koenig \& Roshko 1985), further experimental studies are necessary. The advantage of this approach is that it is easier to develop turbulence models applicable in thin layers similar to the mixing and boundary layers in our theory, than models applicable to the entire separated flow.

Also, other qualitative results and suggestions, such as the unavoidable adverse pressure gradient on the wall of a non-circular cavity, or the advantage of suction over injection for preventing secondary separation, may be relevant to realistic flows.

Various stages of the research described in this publication were made possible in part by Grant No. MK4000 and Grant No. MK4300 from the International Science Foundation and by grants from the Russian Foundation for Fundamental Research, project No. 93-01-17622 and project No. 96-01-01291. The work was completed while one of us (S.C.) was visiting the University of Manchester, with financial support from the Royal Society, which is therefore gratefully acknowledged. We are grateful to Professor N. B. Il'inskii for kind permission to use the code POLET in our research. Special thanks are due to Dr P. Duck for his help in improving the text of the paper. 


\section{REFERENCES}

BATCHELOR, G. K. 1956 A proposal concerning laminar wakes behind bluff bodies at large Reynolds number. J. Fluid Mech. 1, 388-398.

Bunyakin, A. V., Chernyshenko, S. I. \& Stepanov G. Yu. 1996 Inviscid Batchelor-model flow past an aerofoil with a vortex trapped in a cavity. J. Fluid Mech. 323, 367-376.

Chernyshenko, S. I. 1982 An approximate method of determining the vorticity in the separation region as the viscosity tends to zero. Fluid Dyn. 17 (1), 1-11 (translated from Izv. Akad. Nauk SSSR, Mekh. Zhidk. i Gaza No. 2, 40-45).

Chernyshenko, S. I. 1984 Calculation of low-viscosity flows with separation by means of Batchelor's model. Fluid Dyn. 19 (2), 206-210 (translated from Izv. Akad. Nauk SSSR, Mekh. Zhidk. Gaza No. 2, 40-45).

Chernyshenko, S. I. 1988 The asymptotic form of the stationary separated circumference of a body at high Reynolds number. Appl. Math. Mech. 52, 746 (translated from Prikl. Matem. Mekh. 52 (6), 958-966).

Chernyshenko, S. I. 1991 Separated flow over a backward-facing step whose height is much greater than the thickness of the lower sublayer of the interaction zone. Fluid Dyn. 26 (4), 496-501 (translated from Izv. Akad. Nauk SSSR, Mekh. Zhidk. i Gaza No. 4, 25-30).

Cole, J. D. 1968 Perturbation Methods in Applied Mathematics. Waltham (Mass.): Blaisdell.

Herwig, H. 1982 Die Anwendung der asymptotischen Theorie auf laminare Stromungen mit endlichen Ablosegebieter. Z. Flugwiss. Weltzaumforsh. B46, (4), 266-279 (in German).

Koenig, K. \& Roshko, A. 1985 An experimental study of geometrical effects on the drag and flow field of two bluff bodies separated by a gap. J. Fluid Mech. 156, 167-204.

Kolosov, B. V. \& Shifrin, È. G. 1975 On a boundary value problem in a study of closed steady separation region in an incompressible fluid. Prikl. Matem. i Mekh. 39, No. 5, 773-779 (in Russian: English translation in Appl. Math. Mech.).

KrupPA, E. W. 1977 A wind tunnel investigation of the Kasper vortex concept. AIAA Paper 77-310.

Kuethe A. M. \& Chow Ch.-Y. 1986 Foundations of Aerodynamics. John Wiley \& Sons.

Matveeva, N. S. \& Neiland, V. YA. 1967 Laminar boundary layer near a corner point of a body surface. Izv. Akad. Nauk SSSR, Mekh. Zhidk. i Gaza No. 4, 64-70 (in Russian: English translation in Fluid Dyn.).

NeILAND, V. YA. \& SyCHEV V. V. 1966 Asymptotic solutions of the Navier-Stokes equations in regions with large local perturbations. Izv. Akad. Nauk SSSR, Mekh. Zhidk. i Gaza No. 4, 43-49 (in Russian: English translation in Fluid Dyn.).

Ruban, A. I. 1974 On laminar separation from a corner point on a rigid surface. Uch. Zap. TsAGI 5, No. 2, 44-54 (in Russian).

Ruban, A. I. 1976 On the theory of laminar separation from a corner point on a rigid surface. Uch. Zap. TsAGI 7, No. 4, 444-451 (in Russian).

Savitsky, A. I., Schukin, L. N., Karelin, V. G. et al. 1995 Method for control of the boundary layer on the aerodynamic surface of an aircraft, and the aircraft provided with the boundary layer control system. United States Patent No. 5417391, May 23, 1995.

Smith, F. T. 1977 The laminar separation of an incompressible fluid streaming past a smooth surface. Proc. R. Soc. Lond. A 356, 443-463.

SQuire, H. B. 1956 Note on the motion inside a region of recirculation (cavity flow). J. R. Aeron. Soc. 60, 203.

SycheV, V. V. 1972 On laminar separation. Izv. Akad. Nauk SSSR, Mekh. Zhidk. i Gaza No. 3, 47-59 (in Russian: English translation in Fluid Dyn.).

SycheV, V. V. 1978 Boundary layer separation from a flat surface. Uch. Zap. TsAGI 9, No. 3, 20-29 (in Russian).

Sychev, V. V., Ruban A. I., Sychev, Vik. V. \& Korolev, G. L. 1987 Asymptotic Theory of Separated Flows. Moscow: Nauka (in Russian).

Tikhonov, A. N., Goncharski, A. V., Stepanov, V. V. \& Yagoda, A. G. 1990 Numerical Methods for Ill-posed Problems. Moscow: Nauka (in Russian).

WU, J. M. \& WU, J. Z. 1992 Vortex lift at a very high angle of attack with massively separated unsteady flow. In Fluid Dynamic of High Angle of Attack. IUTAM Symp. Tokyo, Japan, September 13-17, 1992 (ed. R. Kawamura \& Y. Aihara), p. 34. Springer. 